ORNL/TM-13413

OAK RIDGE

NATIONAL

LABORATORY

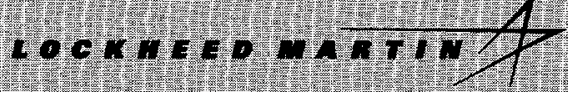

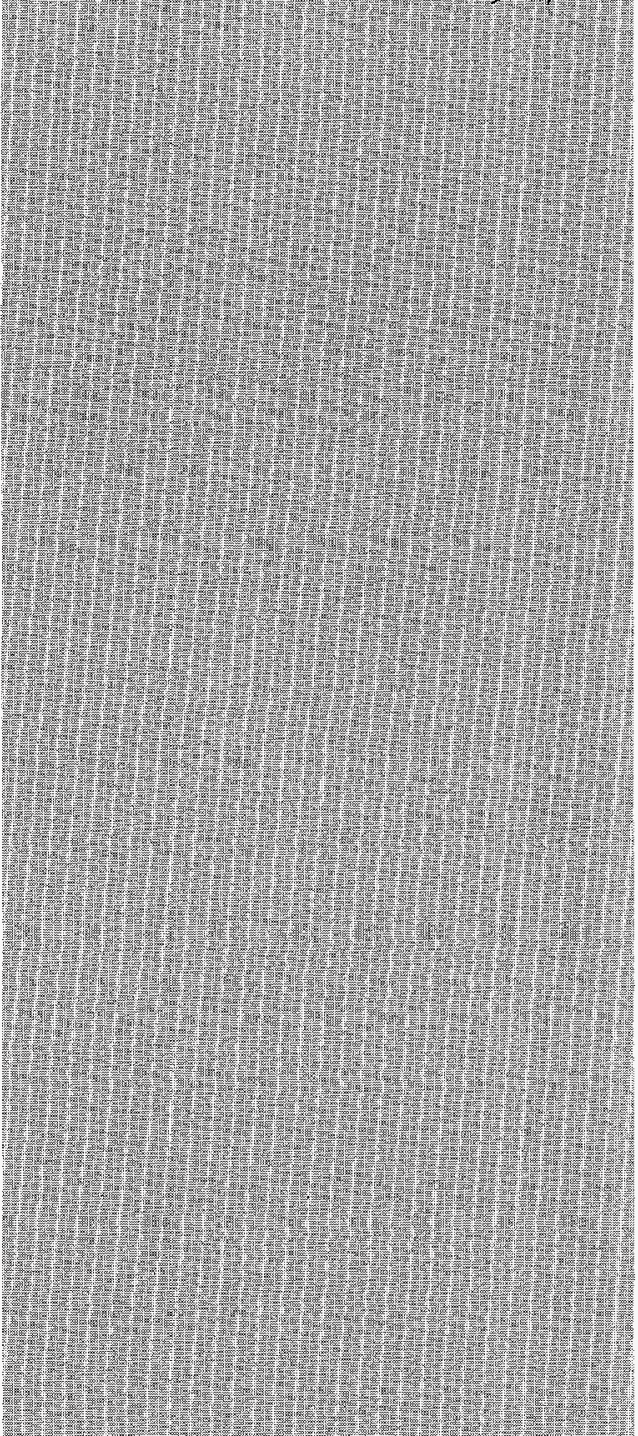

WMAGED AB OPEPTED BY

LOCKHEED 1 IRTH ENEREY RESEARCH CORPORATION FOA THE UATEO STATES

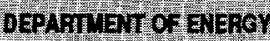

$$
\begin{aligned}
& \text { RECEIVEO } \\
& \text { MAY } 221997 \\
& \text { OSTI }
\end{aligned}
$$

\section{Computing Connection Coefficients of Compactly Supported Wavelets on Bounded Intervals}

C. H. Romine

B. W. Peyton 
This report has been reproduced directly from the best available copy.

Available to DOE and DOE contractors from the Office of Scientific and Technical Information, P. O. Box 62. Oak Ridge. TN 37831: prices available from (423) 576-8401, FTS 626-8401.

Available to the public from the National Technical Information Service, U.S. Department of Commerce, 5285 Port Royal Road. Springfield. VA 22161.

This report was prepared as an account of work sponsored by an agency of the United States Government. Neither the United States Government nor any agency thereof, nor any of their employees, makes any warranty, express or implied, or assumes any legal liability or responsibility for the accuracy, completeness, or usefulness of any information, apparatus, product, or process disclosed, or represents that its use would not infringe privately owned rights. Reference herein to any specific commercial product, process, or service by trade name. trademark, manufacturer, or otherwise, does not necessarily constitute or imply its endorsement, recommendation, or favoring by the United States Government or any agency thereof. The views and opinions of authors expressed herein do not necessarily state or reflect those of the United States Government of any agency thereof. 


\section{DISCLAIMER}

Portions of this document may be illegible electronic image products. Images are produced from the best available original document. 
Computer Science and Mathematics Division

Mathematical Sciences Section

\title{
COMPUTING CONNECTION COEFFICIENTS OF COMPACTLY SUPPORTED WAVELETS ON BOUNDED INTERVALS
}

\author{
C.H. Romine \\ B.W. Peyton \\ Mathematical Sciences Section \\ Oak Ridge National Laboratory \\ P.O. Box 2008, Bldg. 6012 \\ Oak Ridge, TN 37831-6367
}

Date Published: April 1997

Research supported by the Applied Mathematical Sciences subprogram of the Office of Energy Research, U.S. Department of Energy

Prepared by the

Oak Ridge National Laboratory

Oak Ridge, Tennessee 37831

managed by

Lockheed Martin Energy Research Corp.

for the

U.S. DEPARTMENT OF ENERGY

under Contract No. DE-AC05-960R22464 


\section{Contents}

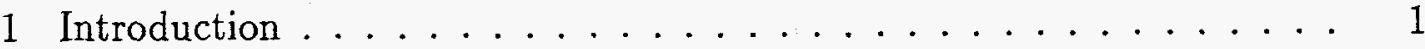

2 Background and Notation .................. 2

3 Computing Proper Connection Coefficients . . . . . . . . . . 7

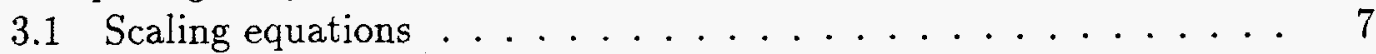

3.2 Moment Equations . . . . . . . . . . . . . . 10

3.3 Normalization Equation . . . . . . . . . . . . 11

4 Results . . . . . . . . . . . . . . . . 13

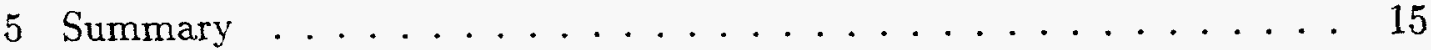

6 Acknowledgements ....................... 15

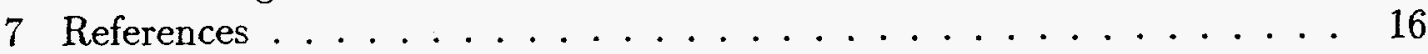

A Tables of Proper Connection Coefficients . . . . . . . . 18 


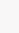




\section{List of Figures}

2.1 The scale function for Daubechies number $N=6 \ldots \ldots \ldots$

2.2 Basis functions for a proper connection coefficient . . . . . . 6

4.1 Illustration of convergence rate $\ldots \ldots \ldots \ldots \ldots$ 



\title{
COMPUTING CONNECTION COEFFICIENTS OF COMPACTLY SUPPORTED WAVELETS ON BOUNDED INTERVALS
}

\author{
C.H. Romine \\ B.W. Peyton
}

\begin{abstract}
Daubechies wavelet basis functions have many properties that make them desirable as a basis for a Galerkin approach to solving PDEs: they are orthogonal, with compact support, and their connection coefficients can be computed. The method developed by Latto et al. [6] to compute connection coefficients does not provide the correct inner product near the endpoints of a bounded interval, making the implementation of boundary conditions problematic. Moreover, the highly oscillatory nature of the wavelet basis functions makes standard numerical quadrature of integrals near the boundary impractical. We extend the method of Latto et al. to construct and solve a linear system of equations whose solution provides the exact computation of the integrals at the boundaries. As a consequence, we provide the correct inner product for wavelet basis functions on a bounded interval.
\end{abstract}




\section{Introduction}

Wavelets are receiving increased attention not only as a mechanism for constructing filter banks or compressing data, but as a natural basis for multilevel schemes for solving PDEs. Several papers in recent years have described the use of wavelet basis functions in solving PDEs, for example Amaratunga et al. [1, 2], Bacry et al. [3], Qian and Weiss [8], and Restrepo and Leaf [9].

Wavelet basis functions have many properties that make them desirable as a basis for a Galerkin approach to solving PDEs: they are orthonormal, with compact support, and their connection coefficients (that is, integrals of products of basis functions, with or without derivatives) can be computed [6]. However, these properties rely on the assumption that the PDE is periodic in the computational domain (which is equivalent to the assumption that the domain is unbounded), and do not all carry over when the domain of the PDE is bounded. Orthogonality, for example, is lost when the basis functions are truncated at a boundary because the domain of integration is a finite interval.

Approaches that assume periodicity complicate the treatment of boundary conditions for PDEs in a finite domain. In a Galerkin formulation, the discretized form of the equation involves connection coefficients on bounded intervals. We call these proper connection coefficients, since they involve proper integrals. The usual connection coefficients computed in Latto et al. with a doubly infinite domain of integration will be called improper connection coefficients. Note that when the support of the integrand lies entirely within the interior of the computational domain, corresponding proper and improper connection coefficients are equal.

The highly oscillatory nature of wavelet basis functions makes standard numerical quadrature for computing connection coefficients impractical. Latto et al. circumvent this problem for improper connection coefficients by exploiting properties of the wavelet basis functions to derive a linear system of equations whose solution has as its components the exact improper connection coefficients.

As far as we know, no one has previously devised a method for computing 
proper connection coefficients. As a result, the natural inner product for Galerkin solution of boundary value problems has been unavailable, and researchers have been restricted to more indirect means of resolving the boundary, e.g., the capacitance matrix method of Proskurowski and Widlund [7]; see Amaratunga, et al. [2] and Qian and Weiss [8]. Motivated by the need to extend methods for resolving boundary conditions in a new and natural direction, we address the problem of computing proper connection coefficients. We adapt the methology of Latto et al., exploiting the properties of the wavelet basis functions to derive two linear systems whose solutions have as their components the exact proper connection coefficients.

The paper is organized as follows. Section 2 presents background and notation. Section 3 describes our technique for computing proper connection coefficients. In section 4 , we use our technique to solve a simple one-dimensional differential equation with Dirichlet boundary conditions. Section 5 provides a few concluding remarks.

\section{Background and Notation}

The first step in developing a basis is to define the underlying scale function. The scale function satisfies the recursive dilation equation

$$
\phi(x)=\sum_{k=0}^{N-1} a_{k} \phi(2 x-k)
$$

where $N$ is an even integer no smaller than two and $\left\{a_{k}\right\}$ are the filter coefficients. Daubechies [5] imposed conditions on the filter coefficients so that the resulting scale functions with Daubechies number $N$ are differentiable and the resulting bases are orthonormal and have $\frac{N}{2}-1$ vanishing moments (i.e., can be used to exactly represent polynomials of degree $\leq \frac{N}{2}-1$ ). We will use all these important properties in our derivations. Throughout we will use the Daubechies scale functions D4 $(N=4), \mathrm{D} 6(N=6), \mathrm{D} 8(N=8)$, etc. A graph of the scale function $\phi$ for $N=6$ is given in Figure 2.1. Also, to improve our notation we 
will let

$$
\phi_{k}(x):=\phi(x-k)
$$

For more about Daubechies wavelets and their properties consult Daubechies [5], Strang [10], Strang and Nguyen [11] or Coddington et al. [4].

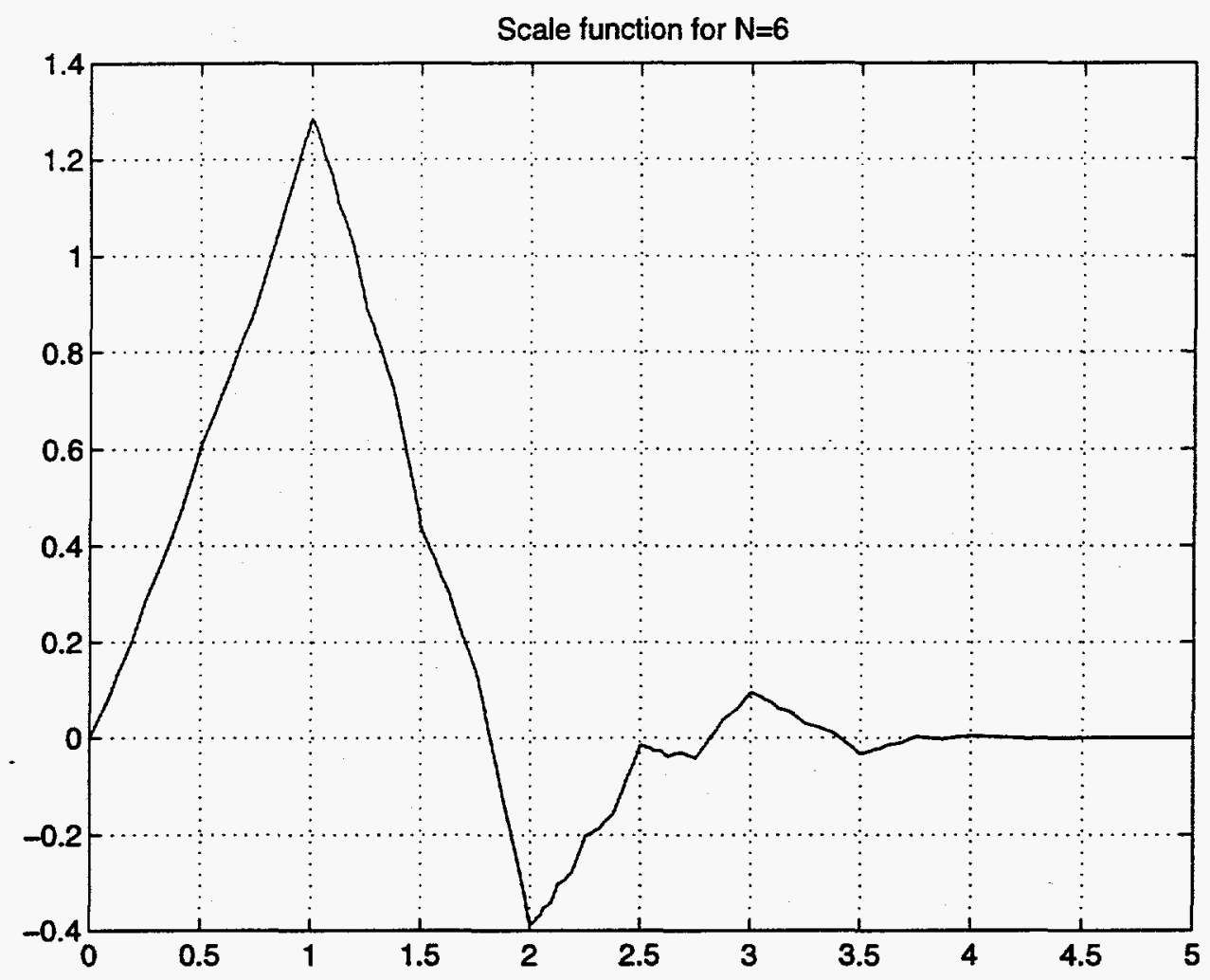

Figure 2.1: The scale function for Daubechies number $N=6$.

The wavelet-Galerkin method for solving PDEs on an unbounded domain produces improper connection coefficients as terms in its equations. If we use the notation $\phi^{(n)}:=\frac{d^{n} \phi}{d x^{n}}$, then the two-term improper connection coefficients are defined (as in Latto, et al.) as

$$
\Lambda_{j}^{d_{1} d_{2}}:=\int_{-\infty}^{\infty} \phi^{\left(d_{1}\right)}(x) \phi_{j}^{\left(d_{2}\right)}(x) d x
$$


and three-term improper connection coefficients are defined by

$$
\Lambda_{j, k}^{d_{1} d_{2} d_{3}}:=\int_{-\infty}^{\infty} \phi^{\left(d_{1}\right)}(x) \phi_{j}^{\left(d_{2}\right)}(x) \phi_{k}^{\left(d_{3}\right)}(x) d x
$$

Only the nonzero coefficients are computed: in the two-term case $2-N \leq j \leq$ $N-2$, and in the three term case $2-N \leq j, k \leq N-2$ and $|j-k| \leq N-2$. There is no loss of generality in fixing the shift on the first term at zero because

$$
\Lambda_{i, j}^{d_{1} d_{2}}=\int_{-\infty}^{\infty} \phi_{i}^{\left(d_{1}\right)}(x) \phi_{j}^{\left(d_{2}\right)}(x) d x=\int_{-\infty}^{\infty} \phi^{\left(d_{1}\right)}(x) \phi_{j-i}^{\left(d_{2}\right)}(x) d x
$$

and

$$
\Lambda_{i, j, k}^{d_{1} d_{2} d_{3}}:=\int_{-\infty}^{\infty} \phi_{i}^{\left(d_{1}\right)}(x) \phi_{j}^{\left(d_{2}\right)}(x) \phi_{k}^{\left(d_{3}\right)}(x) d x=\int_{-\infty}^{\infty} \phi^{\left(d_{1}\right)}(x) \phi_{j-i}^{\left(d_{2}\right)}(x) \phi_{k-i}^{\left(d_{3}\right)}(x) d x
$$

The scale function is the foundation upon which the basis is constructed. Each member of the basis at resolution $m$ is of the form

$$
\phi_{m, k}(x):=2^{m / 2} \phi\left(2^{m} x-k\right)=2^{m / 2} \phi_{k}\left(2^{m} x\right)
$$

Each member of the basis is thus a scaled, dilated, and translated version of the underlying scale function.

The wavelet-Galerkin method for solving PDEs on a bounded domain produces proper connection coefficients as terms in its equations. If we assume (in one dimension) that the interval of computation is $[0,1]$, then for the resolution $m$ basis, the proper two-term connection coefficients will be of the form

$$
\int_{0}^{1} \phi_{m, i}^{\left(d_{1}\right)}(x) \phi_{m, j}^{\left(d_{2}\right)}(x) d x
$$

Since the resolution $m$ basis functions are simply scaled, translated, and dilated versions of the underlying scale function, it is enough to compute proper connec- 
tion coefficients of the form:

$$
\Gamma_{i, j}^{d_{1} d_{2}}:=\int_{0}^{N-1} \phi_{i}^{\left(d_{1}\right)}(x) \phi_{j}^{\left(d_{2}\right)}(x) d x
$$

The limits of integration 0 and $N-1$ are a natural choice to ensure that the support of no basis function in the integrand crosses both limits of integration. Once the proper connection coefficients $\Gamma_{i, j}^{d_{1} d_{2}}$ have been tabulated, all connection coefficients at resolution $m$ can be derived. For example, if the computational domain is $[0,1]$ then assuming $2^{m}>N-1$,

$$
\begin{aligned}
\int_{0}^{1} & \phi_{m,-1}^{\left(d_{1}\right)} \phi_{m,-2}^{\left(d_{2}\right)} d x \\
& \left.=\int_{0}^{2^{m}} \phi^{\left(d_{1}\right)}(y+1) \phi^{\left(d_{2}\right)}(y+2) d y, \text { (where } y=2^{m} x\right) \\
& =\int_{0}^{N-1} \phi^{\left(d_{1}\right)}(y+1) \phi^{\left(d_{2}\right)}(y+2) d y \\
& =\Gamma_{-1,-2}^{d_{1} d_{2}}
\end{aligned}
$$

since the support of $\phi^{\left(d_{1}\right)}(y+1) \phi^{\left(d_{2}\right)}(y+2)$ is [-1, $\left.N-3\right]$. Similarly,

$$
\begin{aligned}
\int_{0}^{1} \phi_{m, 2^{m}-1}^{\left(d_{1}\right)} \phi_{m, 2^{m}-2}^{\left(d_{2}\right)} d x \\
\quad=\int_{-2^{m}+(N-1)}^{N-1} \phi^{\left(d_{1}\right)}(y-(N-2)) \phi^{\left(d_{2}\right)}(y-(N-3)) d y, \\
\quad\left(\text { where } y=2^{m} x-2^{m}+(N-1)\right) \\
=\int_{0}^{N-1} \phi^{\left(d_{1}\right)}(y-(N-2)) \phi^{\left(d_{2}\right)}(y-(N-3)) d y \\
=\Gamma_{N-2, N-3}^{d_{1} d_{2}},
\end{aligned}
$$

since the support of $\phi^{\left(d_{1}\right)}(y-(N-2)) \phi^{\left(d_{2}\right)}(y-(N-3))$ is [N-2,2N-4]. Note that if the support of the integrand lies entirely within the computational domain, the corresponding proper and improper connection coefficients are equal; that is, $\Gamma_{i, j}^{d_{1} d_{2}}=\Lambda_{j-i}^{d_{1} d_{2}}$ (using the notation in Latto, et al.). Two basis functions for a proper two-term connection coefficient that involves a boundary are illustrated in Figure 2.2. 
Three-term proper connection coefficients are defined by

$$
\Gamma_{i, j, k}^{d_{1} d_{2} d_{3}}:=\int_{0}^{N-1} \phi_{i}^{\left(d_{1}\right)}(x) \phi_{j}^{\left(d_{2}\right)}(x) \phi_{k}^{\left(d_{3}\right)}(x) d x
$$

and similar definitions apply for higher numbers of terms. In the two-term case we can restrict our attention to proper connection coefficients for which either $2-N \leq i, j \leq-1$, or $1 \leq i, j \leq N-2$. All others are either zero, or are equivalent to some improper connection coefficient. Similarly, for the three-term case we restrict our attention to proper connection coefficients for which either $2-N \leq i, j, k \leq-1$, or $1 \leq i, j, k \leq N-2$.

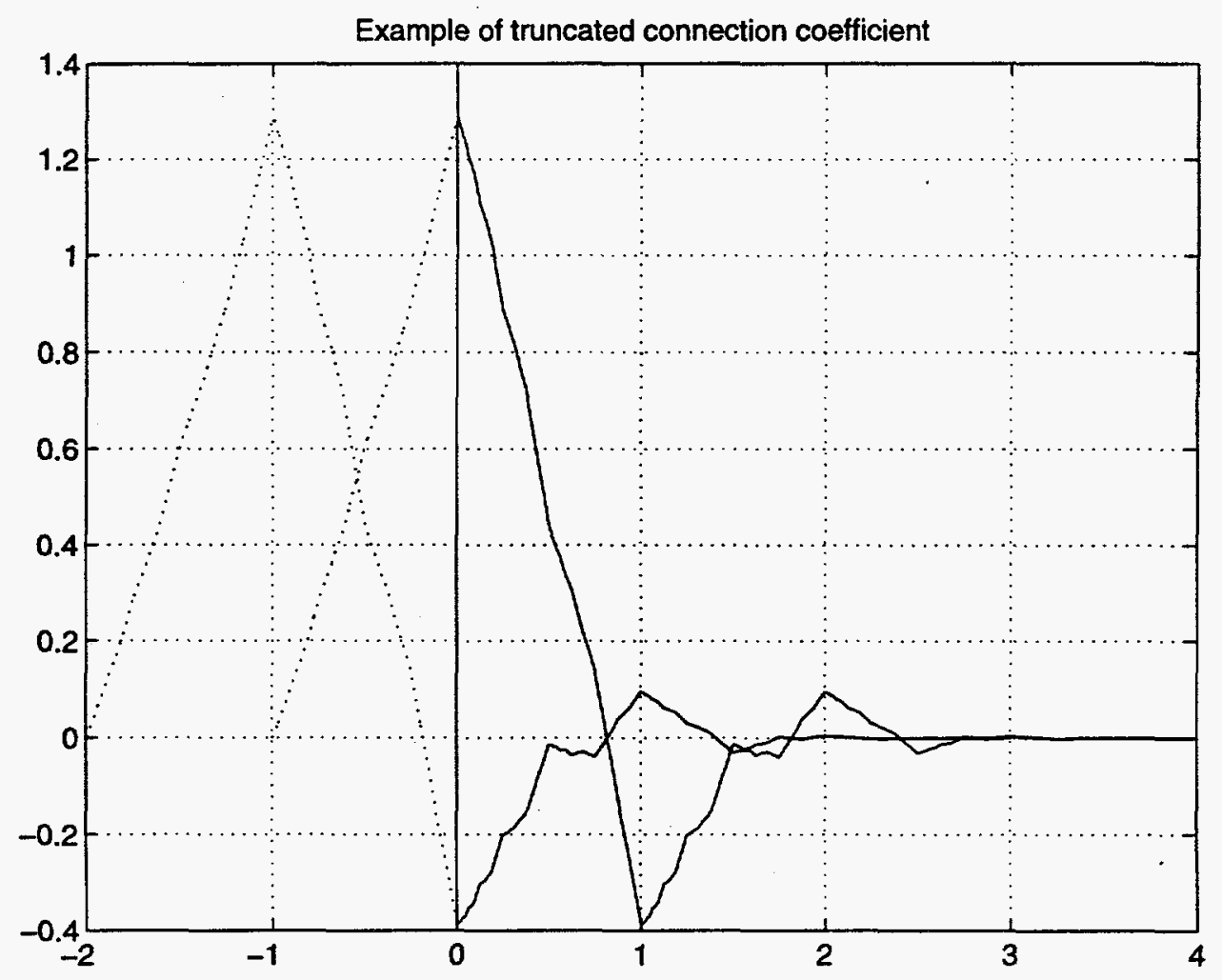

Figure 2.2: Basis functions for a proper connection coefficient

Note that the integrals can no longer be shifted to reduce the total number of distinct proper connection coefficients by assigning a zero shift to the first term. 
Moreover, the truncation of a basis function is not arbitrary, but occurs at one of the dyadic points $\left(i / 2^{m}\right)$ in the given resolution. Equivalently, the truncation occurs at an integer value at the resolution of the scale function. The interval of integration ([0,N-1]) combined with all possible integer shifts cover all possible truncations at integer points of shifted products of the scale function.

\section{Computing Proper Connection Coefficients}

We illustrate our technique by computing the three-term proper connection coefficients. The same technique is easily applied to the two-term proper connection coefficients. Our approach is based on suitable modifications of the scaling equations, moment equations, and the normalization equation, described in Latto, et al. [6].

\subsection{Scaling equations}

We begin this section by deriving a relationship among the unknowns $\Gamma_{i, j, k}^{d_{1} d_{2} d_{3}}$ that will eliminate half of them. We note first that the support of $\phi_{i}^{(d)}$ is $[i, i+(N-1)]$. Then for $1 \leq i, j, k \leq N-2$ we have:

$$
\Gamma_{i, j, k}^{d_{1} d_{2} d_{3}}:=\int_{0}^{N-1} \phi_{i}^{d_{1}} \phi_{j}^{d_{2}} \phi_{k}^{d_{3}} d x
$$

and

$$
\begin{aligned}
\Gamma_{i-(N-1), j-(N-1), k-(N-1)}^{d_{1} d_{2} d_{3}}:=\int_{0}^{N-1} \phi_{i-(N-1)}^{d_{1}} \phi_{j-(N-1)}^{d_{2}} \phi_{k-(N-1)}^{d_{3}} d x \\
=\int_{N-1}^{2 N-2} \phi_{i}^{d_{1}} \phi_{j}^{d_{2}} \phi_{k}^{d_{3}} d x .
\end{aligned}
$$

Hence, since the support of $\phi_{i}^{d_{1}} \phi_{j}^{d_{2}} \phi_{k}^{d_{3}}$ is completely contained in the interval $[0,2 N-2]$, we have the identity

$$
\Gamma_{i, j, k}^{d_{1} d_{2} d_{3}}+\Gamma_{i-(N-1), j-(N-1), k-(N-1)}^{d_{1} d_{2} d_{3}}=\Lambda_{j-i, k-i}^{d_{1} d_{2} d_{3}}
$$


where $\Lambda_{\ell, m}^{d_{1} d_{2} d_{3}}$ denotes the improper connection coefficient described in Latto et al. [6].

We now derive the scaling equations. If $2-N \leq i \leq-1$, then since

$$
\phi_{i}(x)=\phi(x-i)=\sum_{p=0}^{N-1} a_{p} \phi(2 x-(2 i+p))
$$

we have

$$
\phi_{i}^{\left(d_{1}\right)}(x)=2^{d_{1}} \sum_{p=0}^{N-1} a_{p} \phi^{\left(d_{1}\right)}(2 x-(2 i+p)) .
$$

Similarly, with $2-N \leq j, k \leq-1$ we have

$$
\phi_{j}^{\left(d_{2}\right)}(x)=2^{d_{2}} \sum_{q=0}^{N-1} a_{q} \phi^{\left(d_{2}\right)}(2 x-(2 j+q))
$$

and

$$
\phi_{k}^{\left(d_{3}\right)}(x)=2^{d_{3}} \sum_{r=0}^{N-1} a_{r} \phi^{\left(d_{3}\right)}(2 x-(2 k+r))
$$

Hence,

$$
\begin{aligned}
& \Gamma_{i, j, k}^{d_{1} d_{2} d_{3}}=2^{d} \sum_{p=0}^{N-1} \sum_{q=0}^{N-1} \sum_{r=0}^{N-1} a_{p} a_{q} a_{r} \times \\
& \int_{0}^{N-1} \phi^{\left(d_{1}\right)}(2 x-(2 i+p)) \phi^{\left(d_{2}\right)}(2 x-(2 j+q) .) \phi^{\left(d_{3}\right)}(2 x-(2 k+r)) d x
\end{aligned}
$$

(where $d=d_{1}+d_{2}+d_{3}$ ), or

$$
\begin{aligned}
\Gamma_{i, j, k}^{d_{1} d_{2} d_{3}} & =2^{d} \sum_{p=0}^{N-1} \sum_{q=0}^{N-1} \sum_{r=0}^{N-1} a_{p} a_{q} a_{r} \times \\
& \int_{0}^{2 N-2} \phi^{\left(d_{1}\right)}(y-(2 i+p)) \phi^{\left(d_{2}\right)}(y-(2 j+q)) \phi^{\left(d_{3}\right)}(y-(2 k+r)) d y
\end{aligned}
$$


or

$$
\begin{aligned}
& \Gamma_{i, j, k}^{d_{1} d_{2} d_{3}}=2^{d} \sum_{p=0}^{N-1} \sum_{q=0}^{N-1} \sum_{r=0}^{N-1} a_{p} a_{q} a_{r} \times \\
& \quad\left(\int_{0}^{N-1} \phi^{\left(d_{1}\right)}(y-(2 i+p)) \phi^{\left(d_{2}\right)}(y-(2 j+q)) \phi^{\left(d_{3}\right)}(y-(2 k+r)) d y\right. \\
& \quad+\int_{0}^{N-1} \phi^{\left(d_{1}\right)}(y-(2 i+p-(N-1))) \phi^{\left(d_{2}\right)}(y-(2 j+q-(N-1))) \times \\
& \left.\phi^{\left(d_{3}\right)}(y-(2 k+r-(N-1))) d y\right)
\end{aligned}
$$

or

$$
\begin{aligned}
& \Gamma_{i, j, k}^{d_{1} d_{2} d_{3}}=2^{d-1} \sum_{p=0}^{N-1} \sum_{q=0}^{N-1} \sum_{r=0}^{N-1} a_{p} a_{q} a_{r} \times \\
& \left(\Gamma_{2 i+p, 2 j+q, 2 k+r}^{d_{1} d_{2} d_{3}}+\Gamma_{2 i+p-(N-1), 2 j+q-(N-1), 2 k+r-(N-1)}^{d_{1} d_{2} d_{3}}\right)
\end{aligned}
$$

Note that some of the terms on the right hand side of (3.2) are equal to improper connection coefficients, since the entire support of some of the integrands lies within the bounds of integration. These terms are known (thanks to Latto, et. al.), and hence can be moved to the other side. Moreover, we exploit the identity (3.1) to eliminate the unknowns corresponding to $1 \leq i, j, k \leq N-2$, producing a matrix equation of the form:

$$
\left(I-2^{d-1} A\right) \Gamma^{d_{1} d_{2} d_{3}}=R
$$

in $(N-2)^{2}$ unknowns, where $R$ is a vector accumulating the known values in the sum (3.2). A similar treatment holds for the proper connection coefficients $\Gamma_{i, j, k}^{d_{1} d_{2} d_{3}}$ with $1 \leq i, j, k \leq N-2$, though as noted, these can be computed from (3.1) once the values of $\Gamma_{i, j, k}$ for $-(N-2) \leq i, j, k \leq-1$ are known.

The linear system (3.3) is inhomogeneous; however, the coefficient matrix $I-2^{d-1} A$ in (3.3) may not be of full rank. Indeed, our observations of the spectrum of $I-2^{d-1} A$ indicate that it has a zero eigenvalue with multiplicity $d$, 
which would imply that the rank deficiency of the coefficient matrix in (3.3) is $d$.

\subsection{Moment Equations}

If the coefficient matrix in (3.3) is singular, we augment the matrix by adding $d_{1}+d_{2}+d_{3}$ moment equations to the linear system in (3.3), which are derived as follows (see Latto, et al. [6]). We assume that the Daubechies number is sufficiently high that we have $\max \left\{d_{1}, d_{2}, d_{3}\right\}$ vanishing moments. Then for each $q<d_{1}$ there exist coefficients $\left\{M_{i}^{q}\right\}$ such that

$$
x^{q}=\sum_{i=-\infty}^{\infty} M_{i}^{q} \phi_{i}(x)
$$

The set $\left\{M_{i}^{q}\right\}$ are called the moments of $\phi$ and its translates, and the reader is referred to Latto et al. [6] for details on how to compute them.

On the closed interval $[0, N-1]$, we have the identity

$$
x^{q}=\sum_{i=2-N}^{N-2} M_{i}^{q} \phi_{i}(x),
$$

where we have included all terms in the sum whose support intersects the given interval. Differentiating (3.4) $d_{1}$ times yields

$$
0=\sum_{i=2-N}^{N-2} M_{i}^{q} \phi_{i}^{d_{1}}(x)
$$

If we then multiply both sides of (3.5) by $\phi_{j}^{d_{2}} \phi_{k}^{d_{3}}$ for some fixed $j$ and $k$ and integrate over $[0, N-1]$, we obtain

$$
0=\sum_{i=2-N}^{N-2} M_{i}^{q} \Gamma_{i, j, k}^{d_{1} d_{2} d_{3}}
$$

Again, some of the entries on the right hand side of (3.6) are equal to improper connection coefficients, and hence can be moved to the other side. Others can be eliminated via the identity (3.1). This provides $d_{1}$ further (inhomogeneous) 
linear equations in the unknowns $\Gamma_{i, j, k}^{d_{1} d_{2} d_{3}}$, one for each value of $q$. Similarly, we can derive $d_{2}$ further moment equations

$$
0=\sum_{j=2-N}^{N-2} M_{j}^{q} \Gamma_{i, j, k}^{d_{1} d_{2} d_{3}}
$$

and $d_{3}$ moment equations

$$
0=\sum_{k=2-N}^{N-2} M_{k}^{q} \Gamma_{i, j, k}^{d_{1} d_{2} d_{3}}
$$

\subsection{Normalization Equation}

The rectangular system of linear equations derived from the scaling equations and moment equations for the improper connection coefficients is homogeneous, and hence require a nonhomogeneous equation to "normalize" the solution [6]. The rectangular system of equations described above for the proper connection coefficients is already nonhomogeneous; however, the matrix may still be rankdeficient. We now derive the normalization equation for the proper connection coefficients, and include it in the system of equations. We conjecture (and our tests indicate) that the rectangular system of equations containing the scaling equations, the moment equations and the normalization equation is of full rank, and therefore the nonhomogeneous linear system has a unique solution.

If we assume that the basis functions have $\max \left\{d_{1}, d_{2}, d_{3}\right\}$ vanishing moments, then we have the following:

$$
x^{d_{1}}=\sum_{i=-\infty}^{\infty} M_{i}^{d_{1}} \phi_{i}
$$

Differentiating $d_{1}$ times, we obtain

$$
d_{1} !=\sum_{i=-\infty}^{\infty} M_{i}^{d_{1}} \phi_{i}^{d_{1}}
$$

Including only those basis functions whose support intersects the interval [0, $N-1]$, 
we have the identity:

$$
d_{1} !=\sum_{i=2-N}^{N-2} M_{i}^{d_{1}} \phi_{i}^{d_{1}} \text { on }[0, N-1]
$$

Similarly,

$$
d_{2} !=\sum_{j=2-N}^{N-2} M_{j}^{d_{2}} \phi_{j}^{d_{2}} \text { on }[0, N-1]
$$

and

$$
d_{3} !=\sum_{k=2-N}^{N-2} M_{k}^{d_{3}} \phi_{k}^{d_{3}} \text { on }[0, N-1] .
$$

Multiplying equations $(3.7),(3.8)$ and (3.9) together, and integrating over [0,N-1] we obtain

$$
(N-1) d_{1} ! d_{2} ! d_{3} !=\sum_{i=2-N}^{N-2} \sum_{j=2-N}^{N-2} \sum_{k=2-N}^{N-2} M_{i}^{d_{1}} M_{j}^{d_{2}} M_{k}^{d_{3}} \Gamma_{i, j, k}^{d_{1} d_{2} d_{3}}
$$

Again, some of the terms in (3.10) are either known, or can be eliminated via the identity (3.1).

We provide tables of proper connection coefficients in the appendix for the two cases $\left(N=6, d_{1}=0, d_{2}=2\right)$ and $\left(N=6, d_{1}=1, d_{2}=0\right.$ and $\left.d_{3}=0\right)$, the same two cases provided by Latto et al. in [6]. Although the values of $\Gamma_{i, j, k}^{d_{1} d_{2} d_{3}}$ for $1 \leq i, j, k \leq N-2$ can be computed indirectly using the identity (3.1) we compute both tables of proper connection coefficients independently and use 3.1 as an accuracy check. For the tables provided in the appendix, the identity (3.1) is satisfied to within approximately $10^{-15}$.

Since the multiresolution wavelet basis $\left\{\psi_{m, k}\right\}$ is defined in terms of the scale function, the proper connection coefficients derived here can also be used for multiresolution analysis. Specifically, if the multiresolution wavelet basis is used in a Galerkin formulation for the solution of PDEs, the necessary wavelet connection coefficients can be computed directly from the proper connection coefficients given here. 


\section{Results}

In this section, we demonstrate the applicability of proper connection coefficients by solving a simple one-dimensional differential equation on the bounded domain $[0,1]$. We use the 1-D Poisson problem

$$
u_{x x}-f=0
$$

and impose Dirichlet boundary conditions at the endpoints:

$$
u(0)=\alpha \text { and } u(1)=\beta
$$

The Galerkin approach approximates $u$ and $f$ with linear combinations of basis functions:

$$
u \approx \sum_{i} u_{i} \phi_{m, i}, \text { and } f \approx \sum_{i} f_{i} \phi_{m, i}
$$

The left hand side of (4.1) is now approximated by

$$
u_{x x}-f \approx \sum u_{i} \phi_{m, i}^{\prime \prime}-\sum f_{i} \phi_{m, i}
$$

In general, these approximations will not satisfy the differential equation exactly; however, we can find the orthogonal projection onto the space spanned by $\left\{\eta_{j}\right\}$ by ensuring that (4.2) is orthogonal to each so-called "test function" $\eta_{j}$. That is, we define an inner product

$$
\left\langle\phi_{m, i}, \eta_{j}\right\rangle=\int_{0}^{1} \phi_{m, i} \eta_{j} d x
$$

and solve the following linear equations for the unknowns $u_{i}$ :

$$
\sum u_{i}\left\langle\phi_{i}^{\prime \prime}, \eta_{j}\right\rangle-f_{i}\left\langle\phi_{i}, \eta_{j}\right\rangle=0
$$

If the test functions and the basis functions coincide (the choice for Galerkin test functions), the linear system will have a square coefficient matrix. 
One approach to imposing Dirichlet boundary conditions in a Galerkin formulation is to replace two of the rows in (4.3) with the two linear equations derived from the two boundary conditions, preserving a square coefficient matrix. An alternative, and the approach we chose, is to append the two boundary equations to the matrix, and solve the resulting rectangular system.

Our test problem is

$$
u_{x x}=-4 \pi^{2} \sin (2 \pi x), \quad u(0)=1, \quad u(1)=2
$$

whose exact solution is $u=\sin (2 \pi x)+x+1$. Figure 4.1 is a graph of the error as a function of resolution on a $\log -\log$ scale for Daubechies number $N=6$. The slope of the resulting line demonstrates cubic convergence with increase in resolution.

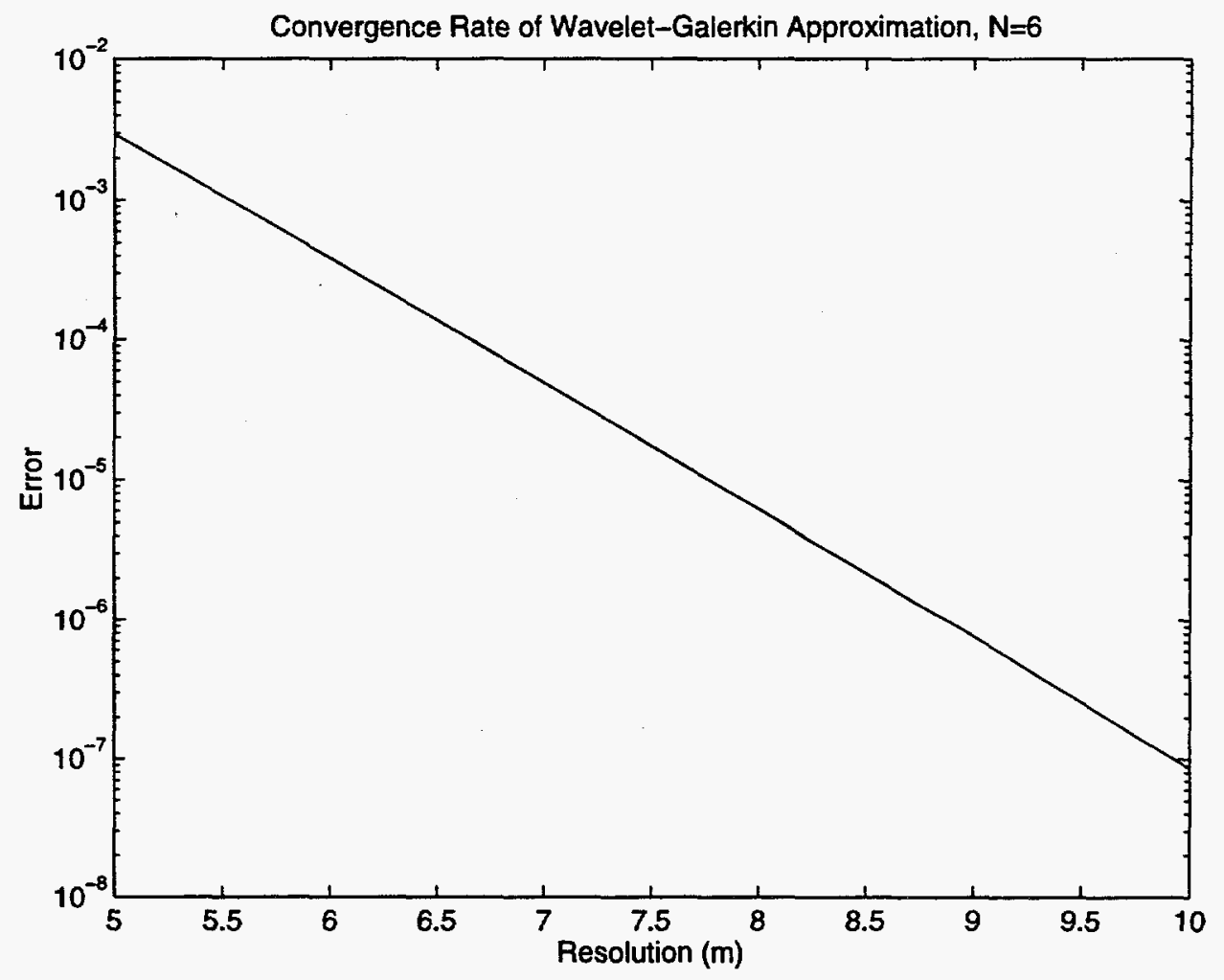

Figure 4.1: Illustration of convergence rate 


\section{Summary}

Proper connection coefficients are important for the solution of nonperiodic PDEs. We have demonstrated a technique for deriving a linear system whose solution is the set of proper connection coefficients needed to compute the natural inner product on bounded intervals. The ability to compute proper connection coefficients provides a natural mechanism for imposing boundary conditions. We exhibited a simple one-dimensional test problem that illustrates the use of proper connection coefficients for PDE's on bounded domains with Dirichlet boundary conditions. We showed that convergence of the solution using Daubechies D6 basis functions was cubic with increasing resolution.

The wavelet-Galerkin approach for solving PDEs has suffered from the inability to properly set PDE problems on bounded domains and to impose boundary conditions in a straightforward way. We have shown that this drawback can be eliminated when the proper connection coefficients can be computed. Moreover, these proper connection coefficients can also be used to compute the proper wavelet connection coefficients arising from a multiresolution analysis of PDEs.

\section{Acknowledgements}

We are grateful to John Drake and Bill Lawkins for our introduction to wavelets for solving PDEs and for many helpful conversations. 


\section{References}

[1] K. Amaratunga and J. Williams, Wavelet based Green's function approach to $2 D P D E$ 's. Unpublished Manuscript.

[2] K. Amaratunga, J. Williams, S. QIAN, AND J. Weiss, WaveletGalerkin solutions for one dimensional partial differential equations, Journal on Numerical Methods in Eng., 37 (1994), pp. 2703-2716.

[3] E. Bacry, S. Mallat, and G. Papanicolaou, A wavelet based spacetime adaptive numerical method for partial differential equations, Mathematical Modelling and Numerical Analysis, 26 (1992), pp. 793-834.

[4] M. Coddington, J. Drake, and W. Shelton, Fast wavelet solutions for the Helmholtz equation. Unpublished Manuscript.

[5] I. DAUBECHIES, Orthonormal bases of compactly supported wavelets, Pure Appl. Math., 41 (1988), pp. 906-966.

[6] A. LatTo, H. Resnikoff, And E. Tenenbaum, The evaluation of connection coefficients of compactly supported wavelets, in Proceedings of the French-USA Workshop on Wavelets and Turbulence, Princeton, New York, 1991, Springer-Verlag.

[7] W. PROSKUROWSKI AND O. WIDLUND, On the numerical solution of Helmholtz's equation by the capacitance matrix method, Math. Comp., 30 (1976), pp. 433-468.

[8] S. QIAN AND J. WEISS, Wavelets and the numerical solution of partial differential equations, Journal of Computational Physics, 106 (1993), pp. 155175.

[9] J. RESTREPO AND G. LEAF, Wavelet-Galerkin discretization of hyperbolic equations, Journal of Computational Physics, 122 (1995), pp. 118-128. 
[10] G. STRANG, Wavelets and dilation equations: A brief introduction, SIAM Review, 31 (1989), pp. 614-627.

[11] G. Strang and T. Nguyen, Wavelets and Filter Banks, WellesleyCambridge Press, Wellesley, MA, 1996. 


\section{A. Tables of Proper Connection Coefficients}

\begin{tabular}{r|rrrr}
\hline & $j=-4$ & \multicolumn{1}{c}{-3} & \multicolumn{1}{c}{-1} \\
\hline$i=-4$ & -0.00038529074526 & -0.00386035962858 & 0.01423373435011 & -0.01534522683341 \\
-3 & -0.00573652265285 & -0.09863430110098 & 0.33947817016054 & -0.35475020354956 \\
-2 & 0.02181281253982 & 0.33904059142268 & -1.37506723951242 & 1.77076145459755 \\
-1 & -0.06519463330387 & -0.90634814610999 & 4.01066114451254 & -5.67304693652727 \\
\hline
\end{tabular}

Table A.1: Table of $\Gamma_{i, j}^{02}:=\int_{0}^{N-1} \phi_{i}(x) \phi_{j}^{(2)}(x) d x$ for $N=6$ 


\begin{tabular}{|c|c|c|c|c|}
\hline \multicolumn{5}{|c|}{$i=-4$} \\
\hline & $k=-4$ & -3 & -2 & -1 \\
\hline$j=-4$ & -0.00000002530682 & -0.00000068992720 & 0.00000306963647 & -0.00001055718019 \\
\hline-3 & -0.00000068992720 & -0.00002337260152 & 0.00010672670559 & -0.00037345654025 \\
\hline-2 & 0.00000306963647 & 0.00010672670559 & -0.00048341871765 & 0.00168470385967 \\
\hline-1 & -0.00001055718019 & -0.00037345654025 & 0.00168470385967 & -0.00585973132675 \\
\hline \multicolumn{5}{|c|}{$i=-3$} \\
\hline & $k=-4$ & -3 & -2 & -1 \\
\hline$j=-4$ & -0.00000032826247 & -0.00000752895920 & 0.00003138211915 & -0.00010322628046 \\
\hline-3 & -0.00000752895920 & -0.00028821307592 & 0.00136993458233 & -0.00488523455350 \\
\hline-2 & 0.00003138211915 & 0.00136993458233 & -0.00714394837814 & 0.02677812707263 \\
\hline-1 & -0.00010322628046 & -0.00488523455350 & 0.02677812707263 & -0.10280576120976 \\
\hline \multicolumn{5}{|c|}{$i=-2$} \\
\hline & $k=-4$ & -3 & -2 & -1 \\
\hline$j=-4$ & 0.00000077866139 & 0.00001753615235 & -0.00007347449696 & 0.00024195517558 \\
\hline-3 & 0.00001753615235 & 0.00076195056336 & -0.00351927324338 & 0.01231761138106 \\
\hline-2 & -0.00007347449696 & -0.00351927324338 & 0.01914653677375 & -0.07281061430283 \\
\hline-1 & 0.00024195517558 & 0.01231761138106 & -0.07281061430283 & 0.28689868099225 \\
\hline \multicolumn{5}{|c|}{$i=-1$} \\
\hline & $k=-4$ & -3 & -2 & -1 \\
\hline$j=-4$ & -0.00000194921939 & -0.00004221923539 & 0.00017491280955 & -0.00057333107231 \\
\hline-3 & -0.00004221923539 & -0.00190418619493 & 0.00818703214249 & -0.02741471871558 \\
\hline-2 & 0.00017491280955 & 0.00818703214249 & -0.04587567964823 & 0.17576474944728 \\
\hline-1 & -0.00057333107231 & -0.02741471871558 & 0.17576474944728 & -0.70948149953627 \\
\hline
\end{tabular}

Table A.2: Table of $\Gamma_{i, j, k}^{100}:=\int_{0}^{N-1} \phi_{i}^{(1)}(x) \phi_{j}(x) \phi_{k}(x) d x$ for $N=6$ 


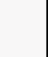

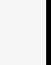


ORNL/TM-13413

INTERNAL DISTRIBUTION

1. B. R. Appleton

2. B. A. Carreras

3. E. F. D'Azevedo

4. T. S. Darland

5. J. M. Donato

6. J. J. Dongarra

7. J. B. Drake

8. T. H. Dunigan

9. G. A. Geist

10. K. L. Kliewer

11. W. F. Lawkins

12-16. M. R. Leuze

17. E. G. $\mathrm{Ng}$
18. C. E. Oliver 19-23. B. W. Peyton 24-28. S. A. Raby

29. B. A. Riley 30-34. C. H. Romine

35. W. A. Shelton

36-40. R. F. Sincovec

41. G. M. Stocks

42. M. R. Strayer

43. P. H. Worley

44. T. Zacharia

45. Central Research Library

46. Laboratory Records - RC

47-48. Laboratory Records Dept.

\section{EXTERNAL DISTRIBUTION}

49. Loyce M. Adams, Applied Mathematics, FS-20, University of Washington, Seattle, WA 98195

50. Christopher R. Anderson, Department of Mathematics, University of California, Los Angeles, CA 90024

51. Todd Arbogast, Department of Mathematical Sciences, Rice University, P. O. Box 1892, Houston, TX 77251

52. Donald M. Austin, 6196 EECS Building, University of Minnesota, 200 Union Street, S.E., Minneapolis, MN 55455

53. Robert G. Babb, Oregon Graduate Center, CSE Department, 19600 N.W. Walker Road, Beaverton, OR 97006

54. David H. Bailey, NASA Ames, Mail Stop 258-5, NASA Ames Research Center, Moffet Field, CA 94035

55. Jesse L. Barlow, Department of Computer Science and Engineering, 220 Pond Laboratory, The Pennsylvania State University, University Park, PA 16802-6106

56. Edward H. Barsis, Computer Science and Mathematics, P. O. Box 5800, Sandia National Laboratory, Albuquerque, NM 87185

57. Adam Beguelin, Carnegie Mellon University, School of Computer Science, 5000 Forbes Avenue, Pittsburgh, PA 15213-3890

58. Robert E. Benner, Parallel Processing Division 1413, Sandia National Laboratories, P. O. Box 5800, Albuquerque, NM 87185

59. Marsha J. Berger, Courant Institute of Mathematical Sciences, 251 Mercer Street, New York, NY 10012 
60. Philippe Berger, Institut National Polytechnique, ENSEEIHT, 2 rue Charles CamichelF, 31071 Toulouse Cedex, France

61. Ake Bjorck, Department of Mathematics, Linkoping University, S-581 83 Linkoping, Sweden

62. John H. Bolstad, L-16, Lawrence Livermore National Laboratory, P. O. Box 808, Livermore, CA 94550

63. Roger W. Brockett, Harvard University, Pierce Hall, 29 Oxford Street Cambridge, MA 02138

64. James C. Browne, Department of Computer Sciences, University of Texas, Austin, TX 78712

65. Bill L. Buzbee, Scientific Computing Division, National Center for Atmospheric Research, P. O. Box 3000, Boulder, CO 80307

66. Donald A. Calahan, Department of Electrical and Computer Engineering, University of Michigan, Ann Arbor, MI 48109

67. Thomas A. Callcott, Director, The Science Alliance Program, 53 Turner House, University of Tennessee, Knoxville, TN 37996

68. Tony Chan, Department of Mathematics, University of California, Los Angeles, 405 Hilgard Avenue, Los Angeles, CA 90024

69. Jagdish Chandra, Army Research Office, P. O. Box 12211, Research Triangle Park, NC 27709

70. Siddhartha Chatterjee, Dept. of Computer Science, CB 3175, Sitterson Hall, The University of North Carolina, Chapel Hill, NC 27599-3175

71. Melvyn Ciment, National Science Foundation, 1800 G Street N.W., Washington, DC 20550

72. Tom Coleman, Department of Computer Science, Cornell University, Ithaca, NY 14853

73. Alva Couch, Department of Computer Science, Tufts University, Medford, MA 02155

74. Paul Concus, Mathematics and Computing, Lawrence Berkeley Laboratory, Berkeley, CA 94720

75. Tom Crockett, ICASE, Mail Stop 132C, NASA Langley Research Center, Hampton, VA 23665-5225

76. Jane K. Cullum, IBM T. J. Watson Research Center, P. O. Box 218, Yorktown Heights, NY 10598

77. George Cybenko, Center for Supercomputing Research and Development, University of Illinois, 104 South Wright Street, Urbana, IL 61801-2932

78. Helen Davis, Computer Science Department, Stanford University, Stanford, CA 94305

79. Michel Dayde, Institut National Polytechnique, ENSEEIHT, 2 rue Charles CamichelF, 31071 Toulouse Cedex, France

80. Craig Douglas, IBM T. J. Watson Research Center, P. O. Box 218, Yorktown Heights, NY 10598-0218 
81. Iain S. Duff, Atlas Centre, Rutherford Appleton Laboratory, Chilton, Oxon OX11 OQX, England

82. Victor Eijkhout, 405 Hilgard Avenue, Department of Mathematics, UCLA, Los Angeles, CA 90024

83. Stanley Eisenstat, Department of Computer Science, Yale University, P. O. Box 2158 Yale Station, New Haven, CT 06520

84. Howard C. Elman, Computer Science Department, University of Maryland, College Park, MD 20742

85. Richard E. Ewing, Director, Institute for Scientific Computations, Texas A\&M University, College Station, TX 77843-3404

86. Edward Felten, Department of Computer Science, University of Washington, Seattle, WA 98195

87. Charles Fineman, Ames Research Center, Mail Stop 269/3, Moffet Field, CA 94035

88. David Fisher, Department of Mathematics, Harvey Mudd College, Claremont, CA 91711

89. Jon Flower, Parasoft Corporation, 2031 S. Myrtle Ave., Monrovia, CA 910164836

90. Geoffrey C. Fox, NPAC, 111 College Place, Syracuse University, Syracuse, NY 13244-4100

91. Chris Fraley, Statistical Sciences, Inc., 1700 Westlake Avenue N, Suite 500, Seattle, WA 98119

92. Joan M. Francioni, Computer Science Department, University of Southwestern Louisiana, Lafayette, LA 70504

93. Paul O. Frederickson, ACL, MS B287, Los Alamos National Laboratory, Los Alamos, NM 87545

94. Offir Frieder, George Mason University, Science and Technology Building, Computer Science Department, 4400 University Drive, Fairfax, Va 22030-4444

95. Robert E. Funderlic, Department of Computer Science, North Carolina State University, Raleigh, NC 27650

96. Dennis B. Gannon, Computer Science Department, Indiana University, Bloomington, IN 47401

97. C. William Gear, NEC Research Institute, 4 Independence Way, Princeton, NJ 08540

98. W. Morven Gentleman, Division of Electrical Engineering, National Research Council, Building M-50, Room 344, Montreal Road, Ottawa, Ontario, Canada K1A 0R8

99. Alan George, Department of Computer Science, University of Waterloo, Waterloo, Ontario, Canada N2L 3G1

100. James Glimm, SUNY-Stony Brook, Department of Applied Mathematics and Statistics, Stony Brook, NY 11794 
101. Gene Golub, Computer Science Department, Stanford University, Stanford, CA 94305

102. Joseph F. Grcar, Division 8245 , Sandia National Laboratories, Livermore, CA 94551-0969

103. William D. Gropp, Mathematics and Computer Science Division, Argonne National Laboratory, 9700 South Cass Avenue, Argonne, IL 60439

104. Eric Grosse, AT\&T Bell Labs 2T-504, Murray Hill, NJ 07974

105. Sanjay Gupta, ICASE, Mail Stop 132C, NASA Langley Research Center, Hampton, VA 23665-5225

106. John L. Gustafson, Ames Laboratory, 236 Wilhelm Hall, Iowa State University, Ames, IA 50011-3020

107. Christian Halloy, Assistant Director of JICS, 104 South College, Joint Institute for Computational Science, University of Tennessee, Knoxville, TN 37996-1301

108. Sven J. Hammarling, The Numerical Algorithms Group, Ltd., Wilkinson House, Jordan Hill Road, Oxford OX2 8DR, United Kingdom

109. Robert M. Haralick, Department of Electrical Engineering, Director, Intelligent Systems Lab, University of Washington, 402 Electrical Engineering Building, FT10, Seattle, WA 98195

110. Ann H. Hayes, Computing and Communications Division, Los Alamos National Laboratory, Los Alamos, NM 87545

111. Michael T. Heath, Department of Computer Science, 2304 Digital Computer Laboratory, University of Illinois, 1304 West Springfield Avenue, Urbanna, IL 618012987

112. Gerald W. Hedstrom, L-71, Lawrence Livermore National Laboratory, P. O. Box 808, Livermore, CA 94550

113. Don E. Heller, Ames Laboratory, 327 Wilhelm, Ames, IA 50011

114. John L. Hennessy, CIS 208, Stanford University, Stanford, CA 94305

115. N. J. Higham, Department of Mathematics, University of Manchester, Gtr Manchester, M13 9PL, England

116. Dan Hitchcock, Office of Scientific Computing, ER-7, Applied Mathematical Sciences, Office of Energy Research, U.S. Department of Energy, Washington, DC 20585

117. Charles J. Holland, Air Force Office of Scientific Research, Building 410, Bolling Air Force Base, Washington, DC 20332

118. Fred Howes, Office of Scientific Computing, ER-7, Applied Mathematical Sciences, Office of Energy Research, Department of Energy, Washington, DC 20585

119. Robert E. Huddleston, Computation Department, Lawrence Livermore National Laboratory, P. O. Box 808, Livermore, CA 94550

120. Jenq-Neng Hwang, Department of Electrical Engineering, FT-10, University of Washington, Seattle, WA 98195

121. Ilse Ipsen, Department of Computer Science, Yale University, P. O. Box 2158 Yale Station, New Haven, CT 06520 
122. Leah H. Jamieson, School of Electrical Engineering, Purdue University, West Lafayette, IN 47907

123. Gary Johnson, Office of Scientific Computing, ER-7, Applied Mathematical Sciences, Office of Energy Research, U.S. Department of Energy, Washington, DC 20585

124. Lennart Johnsson, Thinking Machines Corporation, 245 First Street, Cambridge, MA 02142-1214

125. Harry Jordan, Department of Electrical and Computer Engineering, University of Colorado, Boulder, CO 80309

126. Bo Kagstrom, Institute of Information Processing, University of Umea, 5-901 87 Umea, Sweden

127. Malvyn Kalos, Cornell Theory Center, Engineering and Theory Center Building, Cornell University, Ithaca, NY 14853-3901

128. Hans Kaper, Mathematics and Computer Science Division, Argonne National Laboratory, 9700 South Cass Avenue, Bldg. 221, Argonne, IL 60439

129. Alan H. Karp, HP Labs 3U-7, Hewlett-Packard Company, 1501 Page Mill Road, Palo Alto, CA 94304

130. Linda Kaufman, Bell Laboratories, 600 Mountain Avenue, Murray Hill, NJ 07974

131. Robert J. Kee, Applied Mathematics Division 8245, Sandia National Laboratories, Livermore, CA 94551-0969

132. Kenneth Kennedy, Department of Computer Science, Rice University, P.O. Box 1892, Houston, TX 77001

133. Tom Kitchens, Office of Scientific Computing, ER-7, Applied Mathematical Sciences, Office of Energy Research, U.S. Department of Energy, Washington, DC 20585

134. Clyde P. Kruskal, Department of Computer Science, University of Maryland, College Park, MD 20742

135. Edward Kushner, Intel Corporation, 15201 NW Greenbrier Parkway, Beaverton, OR 97006

136. Michael Langston, Department of Computer Science, University of Tennessee, Knoxville, TN 37996-1301

137. Richard Lau, Office of Naval Research, Code 111MA 800 Quincy Street, Boston Tower 1, Arlington, VA 22217-5000

138. Robert L. Launer, Army Research Office, P. O. Box 12211, Research Triangle Park, NC 27709

139. Tom Leighton, Lab for Computer Science, Massachusetts Institute of Technology, 545 Technology Square, Cambridge, MA 02139

140. Robert Leland, Sandia National Laboratories, 1424, P. O. Box 5800, Albuquerque, NM 87185-5800

141. Randall J. LeVeque, Applied Mathematics, FS-20, University of Washington, Seattle, WA 98195 
142. John G. Lewis, Boeing Computer Services, P. O. Box 24346, M/S 7L-21, Seattle, WA 98124-0346

143. Heather M. Liddell, Center for Parallel Computing, Department of Computer Science and Statistics, Queen Mary College, University of London, Mile End Road, London E1 4NS, England

144. Brent Lindquist, SUNY-Stony Brook, Department of Applied Mathematics and Statistics, Stony Brook, NY 11794

145. Rik Littlefield, Pacific Northwest Laboratory, MS K1-87, P.O.Box 999, Richland, WA 99352

146. Joseph Liu, Department of Computer Science, York University, 4700 Keele Street, Downsview, Ontario, Canada M3J 1P3

147. Franklin Luk, Department of Computer Science, Amos Eaton Building - No. 131 Rensselaer Polytechnic Institute Troy, NY 12180-3590

148. Ewing Lusk, Mathematics and Computer Science Division, Argonne National Laboratory, 9700 South Cass Avenue, MCS 221 Argonne, IL 60439-4844

149. Allen D. Malony, Department of Computer and Information Science, University of Oregon, Eugene, OR 97403

150. Thomas A. Manteuffel, Department of Mathematics, University of Colorado Denver, Denver, CO 80202

151. Anita Mayo, IBM T. J. Watson Research Center, P. O. Box 218, Yorktown Heights, NY 10598

152. Oliver McBryan, University of Colorado at Boulder, Department of Computer Science, Campus Box 425, Boulder, CO 80309-0425

153. James McGraw, Lawrence Livermore National Laboratory, L-306, P. O. Box 808, Livermore, CA 94550

154. Piyush Mehrotra, ICASE, Mail Stop 132C, NASA Langley Research Center, Hampton, VA 23665

155. Paul C. Messina, Mail Code 158-79, California Institute of Technology, $1201 \mathrm{E}$. California Boulevard, Pasadena, CA 91125

156. Cleve B. Moler, MathWorks, 325 Linfield Place, Menlo Park, CA 94025

157. Jorge J. More, Mathematics and Computer Science Division, Argonne National Laboratory, 9700 South Cass Avenue, Argonne, IL 60439

158. William A. Mulder, Koninklijke Shell Exploratie en Produktie Laboratorium, Postbus 60, 2280 AB Rijswijk, The Netherlands

159. David Nelson, Director, Office of Scientific Computing, ER-7, Applied Mathematical Sciences, Office of Energy Research, U.S. Department of Energy, Washington, DC 20585

160. V. E. Oberacker, Department of Physics, Vanderbilt University, Box 1807, Station B, Nashville, TN 37235

161. Dianne P. O'Leary, Computer Science Department, University of Maryland, College Park, MD 20742 
162. Joseph Oliger, Computer Science Department, Stanford University, Stanford, CA 94305

163. James M. Ortega, Department of Computer Science, Thornton Hall, University of Virginia, Charlottesville, VA 22901

164. Steve Otto, Oregon Graduate Institute, Department of Computer Science and Engineering, $19600 \mathrm{NW}$ von Neumann Drive, Beaverton, OR 97006-1999

165. Cherri Pancake, Department of Computer Science, Oregon State University, Corvallis, OR 97331-3202

166. Joseph E. Pasciak, Applied Mathematics, Brookhaven National Laboratory, Upton, NY 11973

167. Merrell Patrick, National Science Foundation, 1800 G Street N.W., Washington, DC 20550

168. Ronald F. Peierls, DAS - Bldg. 490-D, P.O. Box 5000, Brookhaven National Laboratory, Upton, NY 11973

169. Linda R. Petzold, Computer Science Department, University of Minnesota, 200 Union Street, S.E., Room 4-192, Minneapolis, MN 55455

170. Dan Pierce, Boeing Computer Services, P.O. Box 24346, M/S 7L-21, Seattle, WA 98124-0346

171. Robert J. Plemmons, Departments of Mathematics and Computer Science, Box 7311, Wake Forest University, Winston-Salem, NC 27109

172. James C. T. Pool, Deputy Director, Caltech Concurrent Supercomputing Facility, California Institute of Technology, MS 158-79, Pasadena, CA 91125

173. Jesse Poore, Computer Science Department, University of Tennessee, Knoxville, TN 37996-1300

174. David A. Poplawski, Department of Computer Science, Michigan Technological University, Houghton, MI 49931

175. Padma Raghavan, Department of Computer Science, University of Tennessee, Knoxville, TN 37996-1331

176. Daniel A. Reed, Computer Science Department, University of Illinois, Urbana, IL 61801

177. John K. Reid, Numerical Analysis Group, Central Computing Department, Atlas Centre, Rutherford Appleton Laboratory, Didcot, Oxon OX11 0QX, England

178. John R. Rice, Computer Science Department, Purdue University, West Lafayette, IN 47907

179. Garry Rodrigue, Numerical Mathematics Group, Lawrence Livermore National Laboratory, Livermore, CA 94550

180. Donald J. Rose, Department of Computer Science, Duke University, Durham, NC 27706

181. Ahmed H. Sameh, Department of Computer Science, University of Minnesota, 200 Union Street S.E., Minneapolis, MN 55455

182. Joel Saltz, Computer Science Department, A.V. Williams Building, University of Maryland, College Park, MD 20742 
183. Jorge Sanz, IBM Almaden Research Center, Department K53/802, 650 Harry Road, San Jose, CA 95120

184. Robert B. Schnabel, Department of Computer Science, University of Colorado at Boulder, ECOT 7-7 Engineering Center, Campus Box 430, Boulder, CO 803090430

185. Robert Schreiber, Hewlett-Packard Laboratories, 1501 Page Mill Road, M/S 3L-5, Palo Alto, CA 94384-1126

186. Martin H. Schultz, Department of Computer Science, Yale University, P. O. Box 2158 Yale Station, New Haven, CT 06520

187. David S. Scott, Intel Scientific Computers, 15201 N.W. Greenbrier Parkway, Beaverton, OR 97006

188. The Secretary, Department of Computer Science and Statistics, The University of Rhode Island, Kingston, RI 02881

189. Charles L. Seitz, Department of Computer Science, California Institute of Technology, Pasadena, CA 91125

190. Margaret L. Simmons, Computing and Communications Division, Los Alamos National Laboratory, Los Alamos, NM 87545

191. Horst D. Simon, NASA Ames Research Center, Mail Stop T045-1, Moffett Field, CA 94035

192. William C. Skamarock, 3973 Escuela Court, Boulder, CO 80301

193. Tony Skjellum, Dept of Computer Science, Mississippi State University, PO Drawer CS, Mississippi State, MS 39762-5623

194. Burton Smith, Tera Computer Company, 400 North 34th Street, Suite 300, Seattle, WA 98103

195. Marc Snir, IBM T.J. Watson Research Center, Department 420/36-241, P. O. Box 218, Yorktown Heights, NY 10598

196. Larry Snyder, Department of Computer Science and Engineering, FR-35, University of Washington, Seattle, WA 98195

197. Danny C. Sorensen, Department of Mathematical Sciences, Rice University, P. O. Box 1892, Houston, TX 77251

198. Rick Stevens, Mathematics and Computer Science Division, Argonne National Laboratory, 9700 South Cass Avenue, Argonne, IL 60439

199. G. W. Stewart, Computer Science Department, University of Maryland, College Park, MD 20742

200. G. Strang, Department of Mathematics, Massachusetts Institute of Technology, Room 2-240, Cambridge, MA 02139

201. Paul N. Swarztrauber, National Center for Atmospheric Research, P. O. Box 3000, Boulder, CO 80307

202. Julie Swisshelm, Sandia National Laboratories, 1421, Parallel Computational Sciences Department, Albuquerque, New Mexico 87185-5800

203. Wei Pai Tang, Department of Computer Science, University of Waterloo, Waterloo, Ontario, Canada N2l 3G1 
204. Bernard Tourancheau, LIP ENS-Lyon 69364, Lyon cedex 07, France

205. Joseph F. Traub, Department of Computer Science, Columbia University, New York, NY 10027

206. Lloyd N. Trefethen, Department of Computer Science, Cornell University, Ithaca, NY 14853

207. Robert van de Geijn, University of Texas, Department of Computer Sciences, TAI 2.124, Austin, TX 78712

208. Charles Van Loan, Department of Computer Science, Cornell University, Ithaca, NY 14853

209. Udaya B. Vemulapati, Department of Computer Science, University of Central Florida, Orlando, FL 32816-0362

210. Robert G. Voigt, National Science Foundation, Room 417, 1800 G Street N.W., Washington, DC 20550

211. Bi R. Vona, Center for Numerical Analysis, RLM 13.150, University of Texas at Austin, Austin, TX 78712

212. Henk A. van der Vorst, Professor Dept. of Mathematics, Universiteit Utrecht, P.O. Box 80010, 3508 TA, Utrecht, THE NETHERLANDS

213. Michael D. Vose, 107 Ayres Hall, Department of Computer Science, University of Tennessee, Knoxville, TN 37996-1301

214. Phuong Vu, Cray Research, Inc., 19607 Franz Road, Houston, TX 77084

215. A. J. Wathen, School of Mathematics, University Walk, Bristol BSB 1TW, England

216. Robert P. Weaver, 1555 Rockmont Circle, Boulder, CO 80303

217. Mary F. Wheeler, Department of Mathematical Sciences, Rice University, P. O. Box 1892, Houston, TX 77251

218. Andrew B. White, Computing Division, Los Alamos National Laboratory, Los Alamos, NM 87545

219. John Zahorjan, Department of Computer Science and Engineering, Sieg Hall, FR35, University of Washington, Seattle, WA 98195 